\title{
First determination of the dynamical mass of a binary $L$ dwarf ${ }^{\star}$
}

\author{
H. Bouy ${ }^{1,2,4}$, G. Duchêne ${ }^{4,6}$, R. Köhler ${ }^{3}$, W. Brandner ${ }^{3}$, J. Bouvier ${ }^{4}$, E. L. Martín ${ }^{5}$, A. Ghez ${ }^{6}$, X. Delfosse ${ }^{4}$,

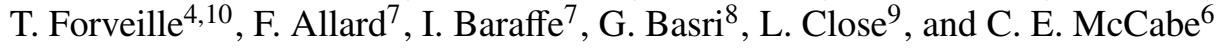 \\ 1 Max Planck für Extraterrestrische Physik, Giessenbachstraße, 85748 Garching bei München, Germany \\ e-mail: hbouy@mpe.mpg.de \\ 2 European Southern Observatory, Karl Schwarzschildstraße 2, 85748 Garching bei München, Germany \\ 3 Max-Planck Institut für Astronomie, Königstuhl 17, 69117 Heidelberg, Germany \\ e-mail: [brandner; koehler] @mpia.de \\ ${ }^{4}$ Laboratoire d'Astrophysique de l'Observatoire de Grenoble, 414 rue de la piscine, 38400 Saint Martin d'Hère, France \\ e-mail: [Jerome. Bouvier;Gaspard.Duchene; Xavier.Delfosse]@obs.ujf-grenoble.fr \\ 5 Instituto de Astrofisica de Canarias, 38200 La Laguna, Spain \\ e-mail: ege@ll.iac.es \\ ${ }^{6}$ Division of Astronomy \& Astrophysics, University of California in Los Angeles, Los Angeles, CA90095-1562, USA \\ e-mail: [ghez;mccabe] @astro.ucla.edu \\ 7 Centre de Recherche Astronomique de Lyon (UML 5574), Ecole Normale Supérieure, 69364 Lyon Cedex 07, France \\ e-mail: [fallard;ibaraffe] @ens-lyon. fr \\ 8 University of California at Berkeley, Astronomy Department, MC 3411 Berkeley, CA 94720, USA \\ e-mail: basri@astro.berkeley.edu \\ 9 Steward Observatory, University of Arizona, 933 N. Cherry Ave., Tucson, AZ 85721-0065, USA \\ e-mail: 1close@as . arizona.edu \\ ${ }^{10}$ Canada-France-Hawaii Telescope Corporation, PO Box 1597, Kamuela Hi-96743, USA \\ e-mail: forveille@cfht.hawaii.edu
}

Received 29 March 2004 / Accepted 11 May 2004

\begin{abstract}
We present here the results of astrometric, photometric and spectroscopic observations leading to the determination of the orbit and dynamical masses of the binary L dwarf 2MASSW J0746425+2000321. High angular resolution observations spread over almost 4 years and obtained with the Hubble Space Telescope (HST), the ESO Very Large Telescope (VLT), and a the W. M. Keck Observatory (Keck) allow us to cover $36 \%$ of the period, corresponding to $60 \%$ of the orbit, and, for the first time, to derive a precise estimate of the total and individual masses of such a late-type object. We find an orbital period of $3850.9_{-767}^{+904}$ days. The corresponding total mass is $0.146_{-0.006}^{+0.016} M_{\odot}$ with uncertainties depending on the distance. Spatially resolved low resolution optical (550-1025 nm) spectra have been obtained with HST/STIS, allowing us to measure the spectral types of the two components ( $\mathrm{L} 0 \pm 0.5$ for the primary and $\mathrm{L} 1.5 \pm 0.5$ for the secondary). We also present precise photometry of the individual components measured on the high angular resolution images obtained with HST/ACS and WFPC2 (visible), $\operatorname{VLT} / \mathrm{NACO}\left(J, H\right.$ and $K_{\mathrm{S}}$ bands) and Keck I ( $K_{\mathrm{S}}$ band). These spectral and photometric measurements enable us to estimate their effective temperatures and mass ratio, and to place the object accurately in a H-R diagram. The binary system is most likely formed by a primary with a mass of $0.085 \pm 0.010 M_{\odot}$ and a secondary with a mass of $0.066 \pm 0.006 M_{\odot}$, thus clearly substellar, for an age of approximately $300 \pm 150 \mathrm{Myr}$. $\mathrm{H} \alpha$ variability indicates chromospheric and/or magnetic activity.
\end{abstract}

Key words. stars: low-mass, brown dwarfs - star: individual: 2MASSW J0746425+2000321 - stars: binary (including multiple): close - techniques: high angular resolution - stars: binaries: visual

\section{Introduction}

With spectral properties intermediate between those of giant planets and late-type stars, ultra-cool and brown dwarfs have opened a new chapter in the study of atmospheric physics and

^ Based on observations obtained with the the NASA/ESA Hubble Space Telescope, the ESO Very Large Telescope (program 70.D-0773), and the W. M. Keck Observatory. chemistry. One of the ultimate goals of a theory of very low mass and sub-stellar objects is an accurate determination of the mass of an object based on spectroscopic characteristics and luminosity. The degeneracy in the mass-luminosity (agetemperature) relation for ultra-cool dwarfs makes it difficult to pin down their physical properties. Luminosities and effective temperatures of ultra-cool dwarfs are function of both age and mass (Burrows et al. 1997) so that an older, slightly 
Table 1. Astrometry and photometry from different catalogues.

\begin{tabular}{|c|c|c|c|c|c|c|c|}
\hline $\begin{array}{l}\text { Date } \\
\text { DD/MM/YYYY }\end{array}$ & $\begin{array}{c}\text { RA } \\
(\mathrm{J} 2000)\end{array}$ & $\begin{array}{c}\text { Dec } \\
(\mathrm{J} 2000)\end{array}$ & Uncert. & Filter & $\begin{array}{l}\text { Mag. } \\
{[\mathrm{mag}]}\end{array}$ & Source & Ident. \\
\hline \multirow[t]{3}{*}{$01 / 01 / 1984^{1}$} & 074642.5 & +200032.6 & $\pm 0 ! 1$ & $R 1$ & 18.28 & USNO-B1.0 & USNO-B1.0 1100-0150847 \\
\hline & & & & $B 2$ & 21.7 & & \\
\hline & & & & $R 2$ & 17.87 & & \\
\hline 01/01/1998 & 074642.55 & +200032.14 & $\pm 0 ! 3$ & $R$ & 17.6 & GSC2.21 & GSC 2W 22110125398 \\
\hline \multirow[t]{3}{*}{$05 / 12 / 1997$} & 074642.56 & +200032.2 & $\pm 0 ! 1$ & $J$ & 11.759 & 2MASS & 2MASSW J07464256+2000321 \\
\hline & & & & $H$ & 11.007 & & \\
\hline & & & & $K$ & 10.468 & & \\
\hline $06 / 12 / 2002$ & & & & $L^{\prime}$ & 11.19 & Leggett et al. (2002) & \\
\hline
\end{tabular}

${ }^{1}$ Mean epoch of observation.

more massive ultra-cool dwarf can exhibit the same effective temperature as a younger, less massive one. Therefore, dynamical masses, which are model-independent, are highly required in order to calibrate the mass-luminosity relation. The study of binary ultra-cool dwarfs offers several advantages for such a study. Both components of the binary are expected to be coeval, thus removing part of the above mentioned degeneracy in the mass-luminosity (age-temperature) relation. Only very few observational constrains on the masses of this class of objects are available nowadays, and we present here the first measurement for field L-dwarfs, at the stellar/substellar transition. The only one similar measurement available up to now concerned the M 8.5/M 9.0 brown dwarf binary GJ 569Bab, presented by Lane et al. (2001). Although the age of 2MASSW J0746425+2000321 is not known independently from any models yet, these observations give promising results as a first step toward the calibration of the models.

In this paper, we will present the results of high angular resolution imaging and low resolution spectroscopic observations of a binary $\mathrm{L}$ dwarf, leading to the determination of its orbital parameters, and total and individual masses. In Sect. 2, we will present 2MASSW J0746425+2000321, in Sect. 3 we will describe the observations and the processing of the data, in Sect. 4 we will explain the calculation of the orbital parameters and in Sect. 5 we will present the analysis of the individual spectra and luminosities and discuss the results.

\section{2MASSW J0746425+2000321}

2MASSW J0746425+2000321 has been observed and reported in several catalogues and articles. It has been identified as a L0.5 dwarf by Kirkpatrick et al. (2000), and suggested to be a binary by Reid et al. (2000) based on its position in a colour-magnitude diagram. It has been resolved as a multiple system by Reid et al. (2001) with a separation of 0.22 and a position angle (PA) of $15^{\circ}$, a measurement later corrected by Bouy et al. (2003) to 0.'219 $\pm 0.0^{\prime} 003$ and PA $=168^{\circ} 8 \pm 0.3$. 2MASSW J0746425+2000321 has been reported in several surveys, such as USNO-B (Monet et al. 2003), GSC2.2, and 2MASS (Cutri et al. 2003). Table 1 gives an overview of the astrometric and photometric properties of 2MASSW J0746425+2000321 as reported in these catalogues. Dahn et al. (2002) and the USNO-B.1 catalogue both report a measurement of the proper motion of this objects,

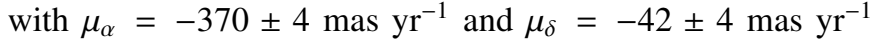

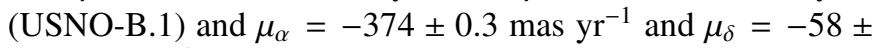

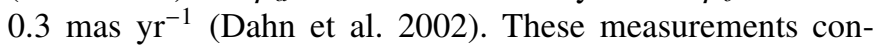
firm that 2MASSW J0746425+2000321 is a common proper motion pair. Such a proper motion indeed implies a motion of $\sim 1$ '. 5 during the 4 years we made the follow up observations, whereas the separation between the two components varied only of $\sim 0$.' 1 . Using high resolution spectra obtained at Keck, Reid et al. (2002) measured a rotational velocity of $24 \mathrm{~km} \mathrm{~s}^{-1}$. Using VLT/UVES high resolution spectra, Bailer-Jones (accepted in A\&A, 2004) measured a rotational velocity ranging between $25.6 \leq v \sin i \leq 30.6 \mathrm{~km} \mathrm{~s}^{-1}$, corresponding to a period between $1.73 \leq T \leq 3.71 \mathrm{~h}$. Both Clarke et al. (2002) and Gelino et al. (2002) report photometric variability, which they attribute mainly to the formation of clouds in the upper layers of the atmospheres. Dahn et al. (2002) measured its distance using trigonometric parallax at $12.21 \pm 0.05 \mathrm{pc}$.

\section{Observation and data processing}

Table 2 gives a log of all the observations we use in this study.

\subsection{High angular resolution imaging with HST/ACS and STIS}

High angular resolution images have been obtained with the HST Advanced Camera for Survey (ACS, Pavlovsky et al. 2003) and Space Telescope Imaging Spectrograph (STIS, Kim Quijano et al. 2003). We observed 2MASSW J0746425+2000321 using the ACS and its High Resolution Channel (HRC) in three different optical filters (F625W, F775W and F850LP), and STIS in the long-pass filter. 2MASSW J0746425+2000321 is clearly resolved on both sets of data (see Fig. 1), and we were able to get precise astrometric and photometric measurements. The data have been analyzed using a custom-made program performing PSF fitting to 
Table 2. Observation log.

\begin{tabular}{|c|c|c|c|c|}
\hline \multicolumn{5}{|c|}{ Imaging } \\
\hline Instrument & Filter & $\begin{array}{c}\text { Exp. } \\
\text { Time [s] }\end{array}$ & $\begin{array}{l}\text { Date obs. } \\
\text { DD/MM/YYYY }\end{array}$ & $\begin{array}{c}\text { Pixel scale } \\
{\left[{ }^{\prime \prime}\right]}\end{array}$ \\
\hline HST/WFPC2-PC & $F 814 W$ & 50 & $25 / 04 / 2000$ & 0.0455 \\
\hline Gemini North/Hokupa & $J$ & 120 & $07 / 02 / 2002$ & 0.'0199 \\
\hline Gemini North/Hokupa & $H$ & 720 & $07 / 02 / 2002$ & 0.'0199 \\
\hline Gemini North/Hokupa & $K$ & 120 & $07 / 02 / 2002$ & 0.'0199 \\
\hline HST/ACS-HRC & F625W & 960 & $21 / 10 / 2002$ & $0^{\prime} 0250^{1}$ \\
\hline HST/ACS-HRC & $F 775 W$ & 440 & $21 / 10 / 2002$ & $0^{\prime} 0250^{1}$ \\
\hline HST/ACS-HRC & $F 850 L P$ & 340 & $21 / 10 / 2002$ & $0.0250^{1}$ \\
\hline VLT/NACO & $K_{\mathrm{S}}$ & 0.4 & $18 / 02 / 2003$ & $0.0148^{2}$ \\
\hline VLT/NACO & $J$ & 10 & $22 / 03 / 2003$ & $0^{\prime} 0148^{2}$ \\
\hline VLT/NACO & $H$ & 5 & $22 / 03 / 2003$ & $0{ }^{\prime} 0148^{2}$ \\
\hline VLT/NACO & $K_{\mathrm{S}}$ & 5 & $22 / 03 / 2003$ & $0 .{ }^{\prime} 0148^{2}$ \\
\hline Keck I/NIRC & $K_{\mathrm{S}}$ & 20 & $04 / 12 / 2003$ & 0.0203 \\
\hline HST/STIS & longpass & 10 & 09/01/2004 & 0.0508 \\
\hline \multicolumn{5}{|c|}{ Spectroscopy } \\
\hline Instrument & $\begin{array}{l}\text { Wavelength } \\
\text { range }[\mathrm{nm}]\end{array}$ & $\begin{array}{c}\text { Exp. } \\
\text { time [s] }\end{array}$ & $\begin{array}{l}\text { Date obs. } \\
\text { DD/MM/YYYY }\end{array}$ & $\begin{array}{c}\text { Dispersion } \\
{[\AA / \text { Aixel }]}\end{array}$ \\
\hline HST/STIS & $525-1300$ & 1980 & $09 / 01 / 2004$ & 4.92 \\
\hline
\end{tabular}

${ }^{1}$ Effective value on the processed images, slightly different from the $0{ }^{\prime} 028 \times 0 .{ }^{\prime} 025$ given in the manual.

${ }^{2}$ Effective value on the processed images, slightly different from the $0 .{ }^{\prime} 01326$ given in the manual.

compute the precise separation, position angle and flux ratios of the multiple system. This program is identical to that used by Bouy et al. (2003), adapted for ACS/HRC and STIS, and is fully described in the mentioned paper. A brief summary is given here for completeness: the PSF fitting routine builds a model binary using ten different PSF stars coming from different ACS/HRC images. A cross-correlation between the model and the binary system yields the best values for the free parameters: separation, position angle and fluxes. The uncertainties and limitations of this technique are discussed in detail in Bouy et al. (2003). The program has been slightly improved since Bouy et al. (2003) presented it in their paper, and the systematic errors and uncertainties on the difference of magnitude are now much better understood and corrected (Bouy 2004). While the original program was using only 5 free parameters (the coordinates of the primary, the coordinates of the secondary, and the flux ratio between the two components), the new version uses 6 free parameters (the 4 coordinates, the flux ratio between the primary and the PSF star, the flux ratio between the secondary and the PSF star), which allows to decrease considerably the systematic errors and uncertainties.

\subsection{High angular resolution imaging with VLT/NACO}

We also obtained high angular resolution images using the ground based facilities offered by ESO on Cerro Paranal on 2003 February 18th and 2003 March 22nd. The VLT on
Yepun uses NACO, an adaptive optics platform (Rousset et al. 2003; Lenzen et al. 2003; Brandner et al. 2002) to achieve diffraction limited images. NACO offers the possibility to use an infrared wavefront sensor, and is therefore ideally suited for the study of ultra-cool and red objects. Its CONICA array offers a $0{ }^{\prime} 01326 \pm 0.001$ pixel-scale that provides critical Nyquist sampling of the diffraction limited images of the telescope at these wavelengths. Its absolute orientation is known to within $\sim 1^{\circ}$.

The atmospheric conditions during the observations were good (respectively $\lambda / r_{0}=0$.' 62 and airmass $=1.5$, and $\lambda / r_{0}=$ 0.'67 and airmass $=1.4$ ), and very sharp images in $K_{\mathrm{S}}$ (first observation) and $J, H$ and $K_{\mathrm{S}}$ (second observation) were obtained with strehl ratios of $S_{r}\left(K_{\mathrm{S}}\right) \sim 30 \%$ (first obs.) and $S_{r}(J) \sim$ $13 \%, S_{r}(H) \sim 27 \%$, and $S_{r}\left(K_{\mathrm{S}}\right) \sim 46 \%$ (last obs.). Figure 1 shows the two $K_{\mathrm{S}}$ images obtained during these two nights.

During the last observation, a PSF star was also acquired in order to perform accurate photometry of the adaptive optics data of the corresponding night. The object, DENIS-P J131500.9-251302 (spectral type $\sim \mathrm{M} 8, J=15.2$, $H=14.54$ and $K_{\mathrm{S}}=14.02 \mathrm{mag}$ ), was observed under better conditions $\left(\lambda / r_{0}=0 . ' 43\right.$ and airmass $\left.=1.03\right)$ with a strehl ratio of $S_{r}(H) \sim 10 \%$, and $S_{r}\left(K_{\mathrm{S}}\right) \sim 40 \%$. Unfortunately it was not observed in $J$. We performed the photometry using standard DAOPHOT PSF fitting photometry. The results are summarized in Table 3. Although the PSF star has a spectral type earlier than 2MASSW J0746425+2000321 and was observed at 


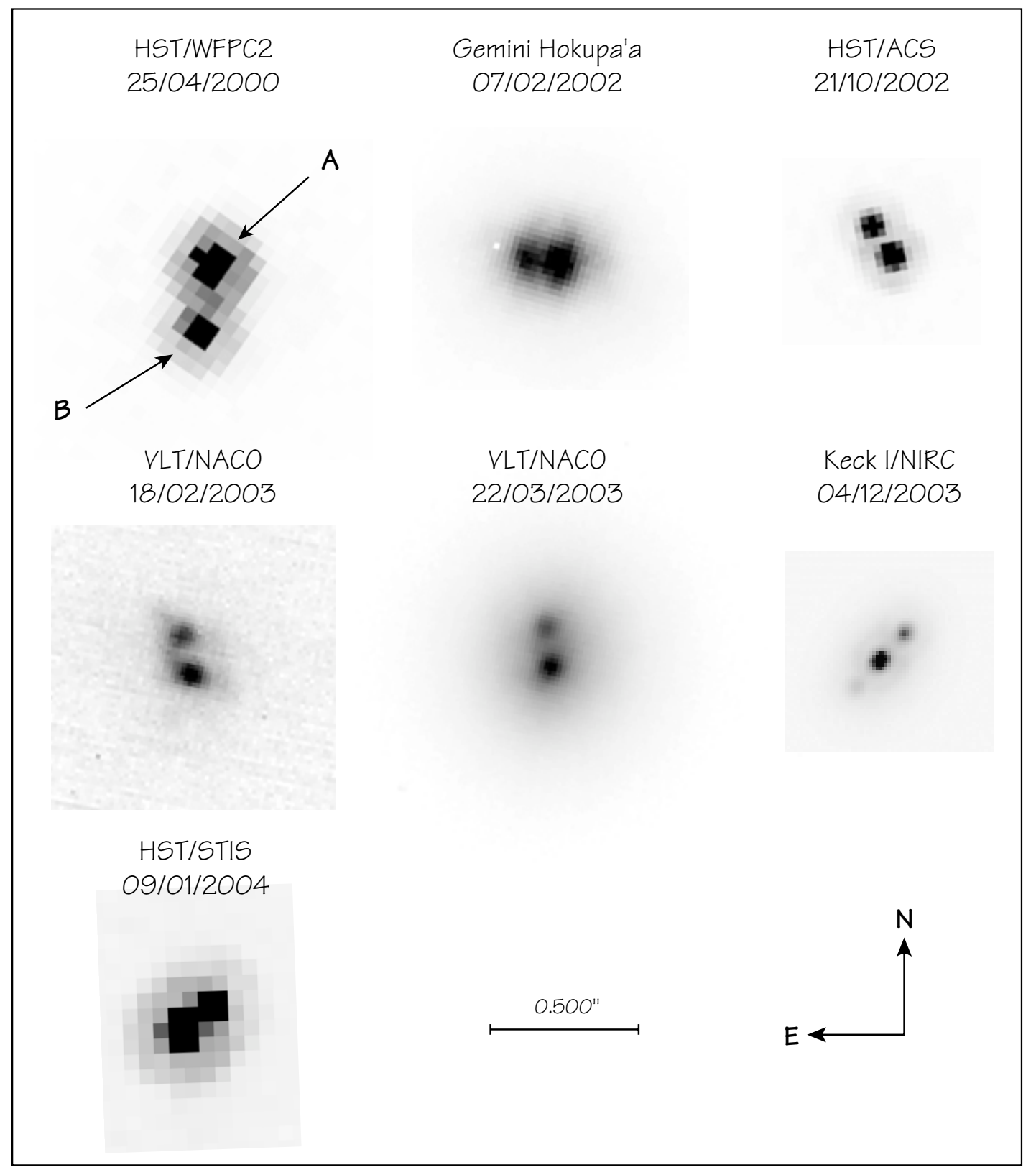

Fig. 1. Images of 2MASSW J0746425+2000321A and B obtained at different epochs with HST, Gemini, NACO and Keck I. The scale and the orientation are the same for all images, and indicated in the figure.

much better airmass and better seeing, the relative photometry we obtain in $H$ and $K_{\mathrm{S}}$ is in very good agreement with the one reported by Close et al. (2003) with Gemini North/Hokupa'a and the one we measure with Keck I/NIRC ( $K_{\mathrm{S}}$ band).

\subsection{Speckle observations with Keck}

On 2003 December 04, we obtained $K$ band speckle observations of our target at the $10 \mathrm{~m}$ Keck I telescope with the facility instrument NIRC (Kleinmann et al. 1994). With its re-imaging optics (Matthews et al. 1996), this $256 \times 256$ nearinfrared array offers a 0 ' $^{\prime} 0203 \pm 0.0003$ pixel scale that provides Nyquist sampling of the diffraction limit of the telescope at this wavelength (about 0. .'05); its absolute orientation is known to within $1^{\circ}$. Several stacks of 200 short-integration exposures were obtained $(t \sim 0.1 \mathrm{~s}$, i.e., fast enough to effectively "freeze" the atmospheric turbulence and retain the high-angular resolution information in the image), and similar stacks on two calibration point sources were obtained immediately before and after our target. Standard speckle data reduction routines were applied to the data; we refer the reader to Ghez (1993) and Patience et al. (1998) for more details and only summarize briefly the various stages involved in the data reduction process. Each individual exposure is first sky subtracted, flat-fielded and bad pixel-corrected; its power spectrum is then calculated. The power spectra are median-averaged over each stack and divided by that of the calibrator. A 2-D sinusoidal function is then fitted to the power spectrum to determine the binary properties: separation, position angle and flux ratios. Uncertainties are 
Table 3. Relative photometry of 2MASSW J0746425+2000321AB.

\begin{tabular}{lccccc}
\hline \hline $\begin{array}{l}\text { Date } \\
\text { DD/MM/YYYY }\end{array}$ & Instrument & Filter & $\begin{array}{c}\text { Mag. Prim. } \\
{[\mathrm{mag}]}\end{array}$ & $\begin{array}{c}\Delta \text { Mag. } \\
{[\mathrm{mag}]}\end{array}$ & $\begin{array}{c}\text { Source } \\
\text { 25/04/2000 }\end{array}$ \\
07/02/2002 & HST/WFPC2 & $F 814 W$ & $15.41 \pm 0.15$ & $1.00 \pm 0.09$ & $(1)$ \\
$07 / 02 / 2002$ & Gemini North/Hokupa'a & $J$ & $12.19 \pm 0.07$ & $0.60 \pm 0.20$ & $(2)$ \\
$07 / 02 / 2002$ & Gemini North/Hokupa'a & $H$ & $11.54 \pm 0.11$ & $0.48 \pm 0.15$ & $(2)$ \\
$21 / 10 / 2002$ & HST/ACS & $F 625 W$ & $18.81 \pm 0.05$ & $0.48 \pm 0.03$ & $(3)$ \\
$21 / 10 / 2002$ & HST/ACS & $F 775 W$ & $15.98 \pm 0.05$ & $0.68 \pm 0.04$ & $(3)$ \\
$21 / 10 / 2002$ & HST/ACS & $F 850 L P$ & $14.24 \pm 0.05$ & $0.76 \pm 0.04$ & $(3)$ \\
$22 / 03 / 2003$ & VLT/NACO & $H$ & $11.55 \pm 0.08$ & $0.46 \pm 0.15$ & $(3)$ \\
$22 / 03 / 2003$ & VLT/NACO & $K_{\mathrm{S}}$ & $11.06 \pm 0.09$ & $0.42 \pm 0.15$ & $(3)$ \\
$04 / 12 / 2003$ & Keck I/NIRC & $K_{\mathrm{S}}$ & $11.03 \pm 0.03$ & $0.52 \pm 0.03$ & $(3)$ \\
\hline
\end{tabular}

${ }^{1}$ Source: (1) Bouy et al. (2003); (2) Close et al. (2003); (3) this paper.

Table 4. Relative astrometry of 2MASSW J0746425+2000321AB.

\begin{tabular}{lccccc}
\hline \hline $\begin{array}{l}\text { Date } \\
\text { DD/Mм/YYYY }\end{array}$ & $\begin{array}{c}\text { Time }^{1} \\
\text { HH/Mm/ss }\end{array}$ & $\begin{array}{c}\text { Sep. }^{2} \\
{[\mathrm{mas}]}\end{array}$ & $\begin{array}{c}\text { PA }^{2} \\
{\left[{ }^{\circ}\right]}\end{array}$ & Instrument & Source $^{3}$ \\
\hline $25 / 04 / 2000$ & $08: 14: 14$ & $219 \pm 3$ & $168.8 \pm 0.8$ & HST/WFPC2 & $(1)$ \\
$07 / 02 / 2002$ & $09: 48: 55$ & $121 \pm 8$ & $85.7 \pm 3.5$ & Gemini North/Hokupa'a & $(2)$ \\
$21 / 10 / 2002$ & $23: 10: 43$ & $119.5 \pm 1$ & $33.9 \pm 0.5$ & HST/ACS & $(3)$ \\
$18 / 02 / 2003$ & $01: 40: 45$ & $131.3 \pm 3.9$ & $13.8 \pm 1.9$ & VLT/NACO & $(3)$ \\
$22 / 03 / 2003$ & $01: 22: 00$ & $123.5 \pm 2.1$ & $4.6 \pm 1.0$ & VLT/NACO & $(3)$ \\
$04 / 12 / 2003$ & $15: 15$ & $126.5 \pm 1.8$ & $317.9 \pm 0.7$ & Keck I/NIRC & $(3)$ \\
$09 / 01 / 2004$ & $18: 51: 45$ & $134.5 \pm 3$ & $311.1 \pm 1.2$ & HST/STIS & $(3)$ \\
\hline
\end{tabular}

${ }^{1}$ The uncertainty corresponds to the exposure time (see Table 2).

${ }^{2} 1-\sigma$ uncertainties (combined instrumental and measurement).

${ }^{3}$ Source: (1) Bouy et al. (2003); (2) Close et al. (2003); (3) this paper.

estimated from the standard deviation of the parameters extracted from all stacks. There is a $180^{\circ}$ ambiguity in the position angle of the binary as derived through power spectrum analysis, but this can be resolved by shift-and-adding all individual exposures using the brightest speckle as a reference. The resulting image shows the companion to be roughly to the Northeast of the primary and the astrometric accuracy of the power spectrum analysis is much higher. The results obtained are reported in Tables 3 and 4 and Fig. 1 shows the final image. As mentioned above, the difference of magnitude is in very good agreement with the previous measurements within the uncertainties.

\subsection{High angular resolution - Low spectral resolution optical spectroscopy with STIS}

In order to get the spatially resolved spectra of each component of the multiple system, we tried to align the slit along the axis of the binary. Scheduling constraints of HST made it difficult to get long slit STIS observations of 2MASSW J0746425+2000321 at a particular roll angle of HST. In order to ease scheduling, a range of admitable roll angle was defined. This combined with the relatively rapid orbital motion of 2MASSW J0746425+2000321 meant that the entrance slit of STIS was not optimally aligned with the position angle of 2MASSW J0746425+2000321 at the time of the observations. Since the size of the slit we used ( 0.2$)$ is larger than the separation of the binary $\left(\sim 0{ }^{\prime} 125\right)$, we could nevertheless obtain a resolved 2-D spectrum and perform an extraction of the two spectra. The effects on the spectral analysis can be the following: since the red and the blue photocentres of each component are not symmetrically centred in the slit, the dispersion of the red and blue parts of the spectrum suffers differently from obstruction by the slit. Since the position of the photocentres and the dispersion of the light depends on the wavelength, the loss in flux also varies with the wavelength. This effect produces a "bluer" spectrum for the secondary.

The separation between the two spectra is about 2 pixels, whereas the full width at half maximum $(F W H M)$ of the line spread function (LSF) varies between 1.0 and 1.2 pixel, so that the two spectra are barely resolved. In order to extract 


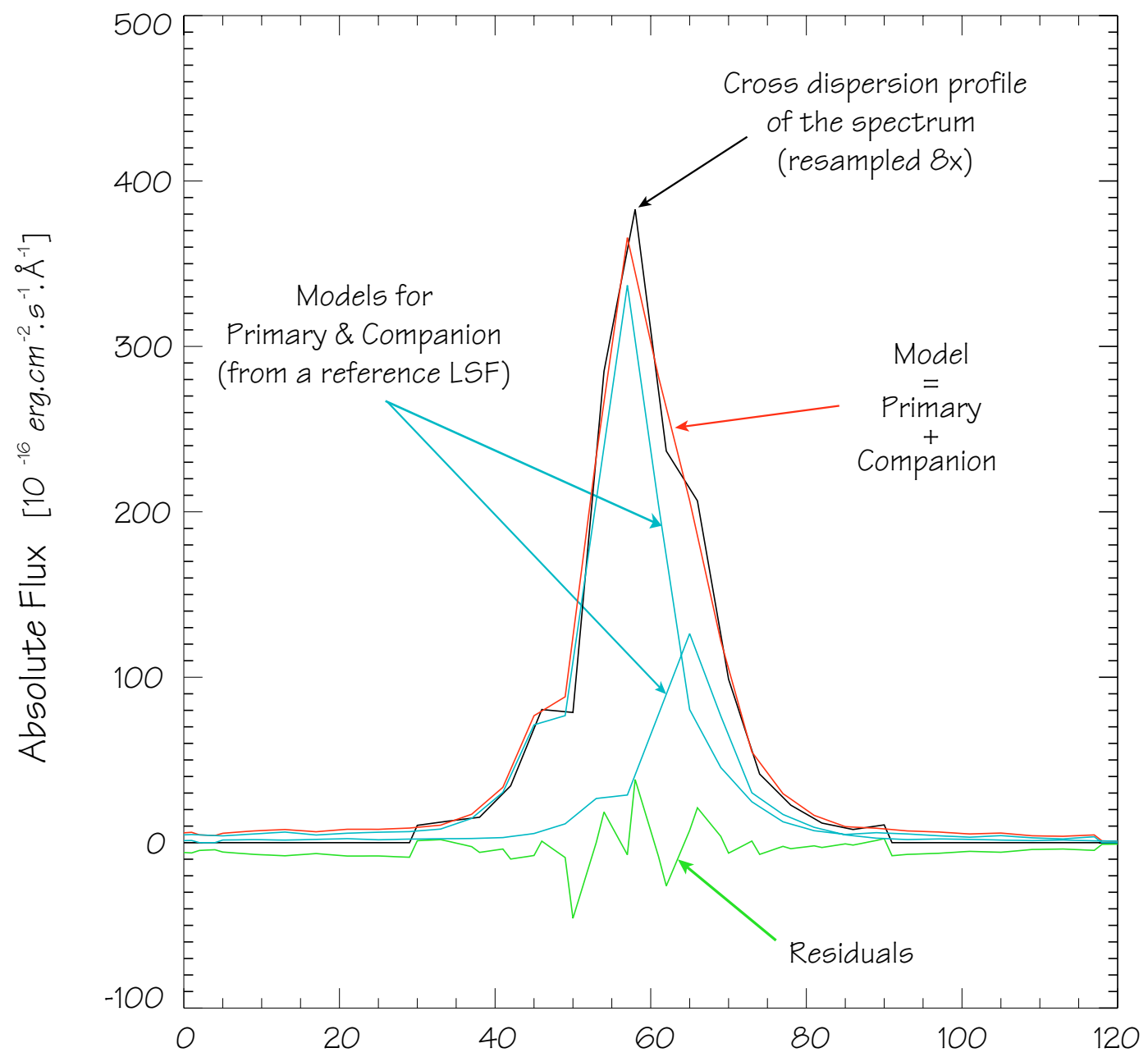

Fig. 2. Extraction of the individual spectra. This figure shows the cross dispersion profile of the 2-D spectrum around $850 \mathrm{~nm}$, and the best fits of the primary and the secondary (light blue). The sum of the primary and secondary (red) is also indicated for comparison with the raw data (black). In that case the intensity of the residuals (green) is less than $\sim 2 \%$ of the intensity of the raw data.

the spectrum of each component, we used a custom made program able to perform a fit of the two blended spectra. On each cross-dispersion column, a minimum $\chi^{2}$ fit was performed to the data using the cross-dispersion profile of a reference spectrum at the same wavelength. The latter spectrum was obtained with the same instrument settings on a K7 dwarf (TWA 6, program 8176 , P. I. Schneider). The free parameters for the fit are the amplitude of the primary, the amplitude of the secondary, the position of the primary and the position of the secondary. Since the cross dispersion profile is barely sampled, we also performed a linear re-sampling of the data by a factor of eight prior to the fit, in order to avoid problems due to spectral aliasing. To ensure more robustness and increase reliability, the program was used in 2 passes. A polynomial fit of the results on the positions of the two individual spectra was made after the first pass, in order to identify and remove outliers (due to bad pixels or cosmic rays). The results of these fits were then used as first guess inputs for the second pass. The results obtained with the second pass are very close to that obtained with the first pass but cleaner (without the more obvious bad pixels, cosmic rays and outliers), ensuring that the whole algorithm is robust enough and converging properly. Figure 2 gives an overview of the results at a particular wavelength. The residuals after the fit represent between $1.5 \%$ and $9 \%$ of the total intensity of the original spectrum depending on the wavelength, thus of the same order than the signal to noise ratio, ensuring that the quality of the fit is good.

\section{Orbital parameters and determination of the total mass}

We used three different and independent custom-made programs to determine the best fitting orbital solution for 2MASSW J0746425+2000321 and the uncertainties on each of the fitted parameters. The orbit can be entirely described by seven independent parameters: semi-major axis $(a)$, orbital period $(P)$, inclination $(i)$, eccentricity $(e)$, position angle of the ascending node $(\Omega)$, angle between the ascending node and periastron $(\omega)$ and time of periastron passage $\left(T_{0}\right)$. With seven two-dimension astrometric data-points, this fully-constrained problem has seven free parameters. The total mass of the system can be derived from the orbital period and semi-major axis through Kepler's Third Law. 


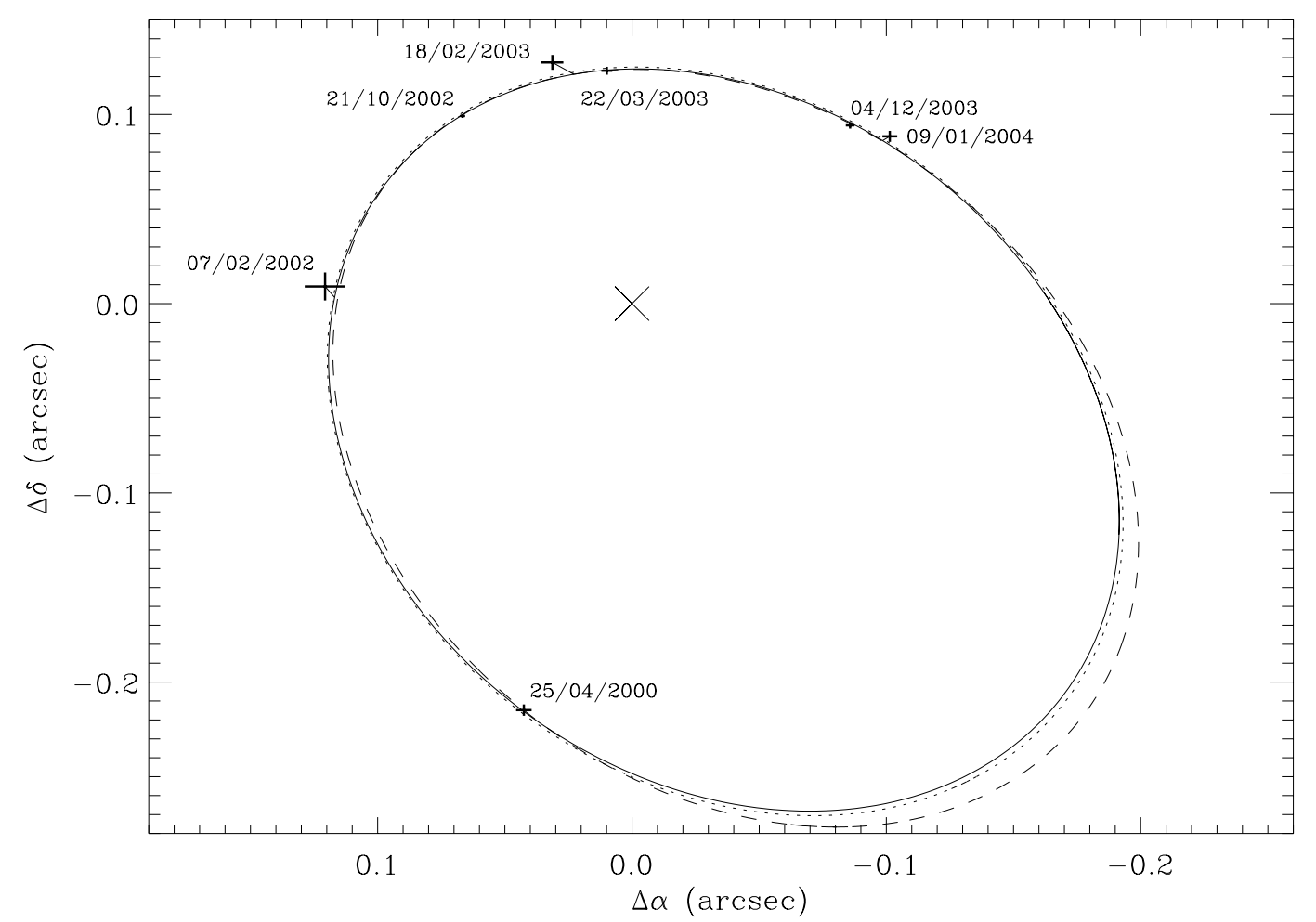

Fig. 3. Positions of 2MASSW J0746425+2000321A and B and best fit of the orbit . The dotted curve represents the best bit orbit obtained with the amoeba method, and the solid curve the result obtained with the iterative method, and the dashed curve the solution given by ORBIT. It appears clearly that the three methods give close results, well within the uncertainties. The plus indicate the observations and their uncertainties, and the corresponding epoch is indicated. The central cross shows the position of the primary.

Table 5. Orbital parameters of 2MASSW J0746425+2000321AB.

\begin{tabular}{lccc}
\hline \hline Parameter & ${\text { Iterative } \text { method }^{1}}^{\text {Amoeba } \text { Method }}$ & ORBIT $^{2}$ \\
\hline Total Mass $\left[M_{\odot}\right]$ & $0.146_{-0.006}^{+0.016}$ & 0.1511 & 0.148 \\
Period, $P$ [days] & $3850.9_{-767}^{+904}$ & 3718 & $3863 \pm 609$ \\
Eccentricity, $e$ & $0.41_{-0.09}^{+0.08}$ & 0.3999 & $0.417 \pm 0.062$ \\
Semi-major axis, $a,[\mathrm{AU}]$ & $2.53_{-0.28}^{+0.37}$ & 2.50 & $2.55 \pm 0.25$ \\
Inclination, $i\left[^{\circ}\right]$ & $141.6_{-3.4}^{+2.5}$ & 141.65 & $140.65 \pm 2.29$ \\
Argument of Periapsis, $\omega\left[^{\circ}\right]$ & $350.6_{-5.9}^{+5.2}$ & 350.15 & $350.65 \pm 3.58$ \\
Longitude of ascending node, $\Omega\left[^{\circ}\right]$ & $20.7_{-14.2}^{+9.9}$ & 18.97 & $20.84 \pm 7.68$ \\
Periastron Passage, $T_{0}($ year $)$ & $2002.89_{-0.09}^{+0.14}$ & 2002.91 & $2002.84 \pm 0.07$ \\
reduced- $\chi^{2}$ of the fit & 1.38 & 1.41 & 1.46 \\
\hline
\end{tabular}

${ }^{1} 2-\sigma$ uncertainties, corresponding to a $95.4 \%$ level of confidence. These uncertainties do not include the uncertainty on the distance $(\sim 2.4 \%$, $2-\sigma)$, which should be added in quadrature.

$2-\sigma$ uncertainties. These uncertainties do include the uncertainty on the distance, but assume that the uncertainty are linear, which is not the case here.

\section{1. "Amoeba" method}

The first method minimizes in the nonlinear 7-dimensions function by downhill simplex method, using the amoeba algorithm (see e.g. Press et al. 1992, for a description of the method and algorithm.). It fits all seven orbital parameters simultaneously, taking into account non-equal errors of the measurements. The reduced- $\chi^{2}$ of 1.41 ensures that the fit is satisfactory (see Sect. 4.4 below). The results are shown in Fig. 3 and Table 5. No uncertainties on the derived parameters are available with this method.

\subsection{Iterative method}

This method uses 50000 independent starting points that consist of a set of the 7 parameters being randomly chosen from their entire range of possible values. For each starting point, a Powell convergence algorithm minimizing the total $\chi^{2}$ 


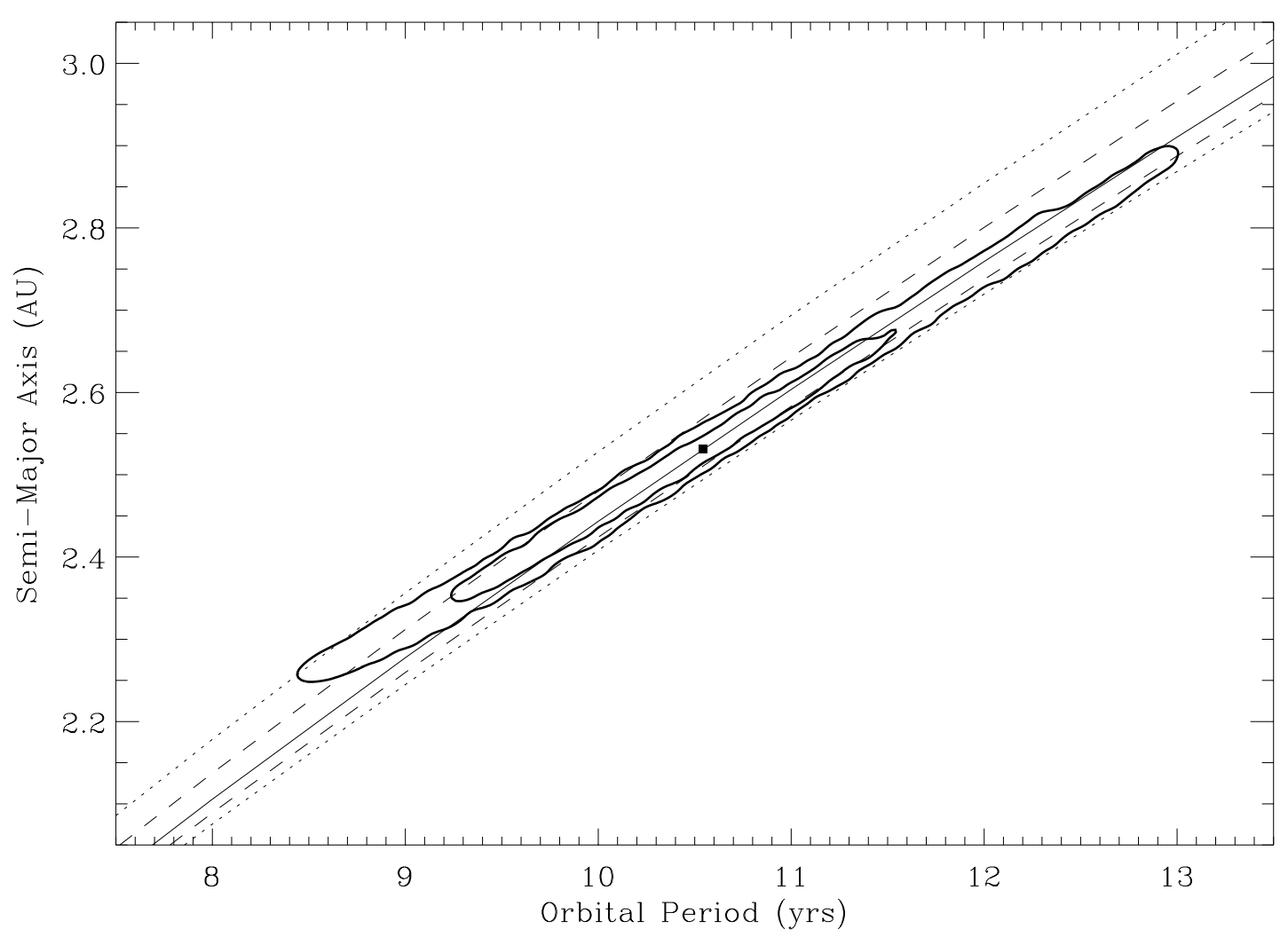

Fig. 4. Ambiguity in the orbital parameters: the contours represent the solutions at 1 - and $2-\sigma\left(\Delta \chi^{2}=1.0\right.$ and 4.0 respectively) in the semi-major axis vs period space. The lines corresponding to different masses indicate the levels of confidence at $68.7 \%$ and $95.4 \%$ (1- and $2-\sigma$ respectively). The filled square in the centre corresponds to the best fit. Although relatively large ranges are possible for the period and the semi-major axis, the range of corresponding acceptable masses is very narrow: between 0.143 and $0.153 M_{\odot}$ at $1-\sigma$ (represented by dashed lines) and between 0.141 and $0.160 M_{\odot}$ at 2- $\sigma$ (dotted lines). Although the orbit is not perfectly known yet, the mass is relatively precisely determined, with a best value at $0.146_{-0.006}^{+0.016} M_{\odot}(2-\sigma$ uncertainties, solid line).

(Press et al. 1992) modifies simultaneously all 7 parameters until it converges to a local minimum. Once convergence for all 50000 sets of initial guesses has been achieved, we read through the output file to find the absolute minimum of the $\chi^{2}$ function, which reveals the best-fitting orbital solution. Our best-fitting solution, illustrated in Fig. 3 has a satisfying reduced $-\chi^{2}$ value of 1.38 .

Uncertainties for each parameters are defined by the range of possible values indicated by all solutions with total $\chi^{2}$ between $\chi_{\min }^{2}$ and $\chi_{\min }^{2}+4$. These represent the $95.4 \%$ confidence level for each parameters. Due to the highly non-linear behavior of the equations of orbital motion, the uncertainties do not follow a Gaussian statistics and nor even symmetric about the best fit. Note that the uncertainties are derived under the assumption that all parameters are independent, which is not correct. For instance, the uncertainties derived for $P$ and $a$ would yield an uncertainty on the system mass on order of $\pm 0.055 M_{\odot}$, $\sim 4$ to 9 times larger than we actually derived here. Therefore, the uncertainties quoted here are only valid if they are used for one parameter at a time. Figure 4 shows that this is because the fitted values of $P$ and $a$ are tightly correlated and correspond to a very narrow range of possible masses. Although the orbit is not perfectly known yet, the total mass is relatively precisely determined: $M_{\text {tot }}=0.146_{-0.006}^{+0.016} M_{\odot}$, corresponding to $4 \sim 11 \%$ uncertainty.
The uncertainty on the distance to the system translates into a separate $2.4 \%$ uncertainty $(2-\sigma)$, or $0.035 M_{\odot}$, on the system mass. The uncertainty on the orbital fitting is therefore the major source of uncertainty for this binary system since both sources of uncertainty (fit and distance) should be added in quadrature.

\subsection{ORBIT}

We also used the ORBIT program of Forveille et al. (1999), fully described in their article. Briefly, "the program performs a least square adjustment to all available observations, with weights inversely proportional to the square of their standard errors [...] The program uses a Levenberg-Marquardt minimization algorithm (Marquardt 1963) [...] Standard errors for derived parameters are computed from the full covariance matrix of the linearized least square adjustment."

\subsection{Reduced- $\chi^{2}$ and uncertainties}

The reduced- $\chi^{2}$ values of $\sim 1.4$ indicate that some of the uncertainties on the astrometric measurements may be slightly underestimated, although with only seven measurements for seven free parameters, such a value is statistically acceptable. Although rescaling the astrometric uncertainties to reach a 

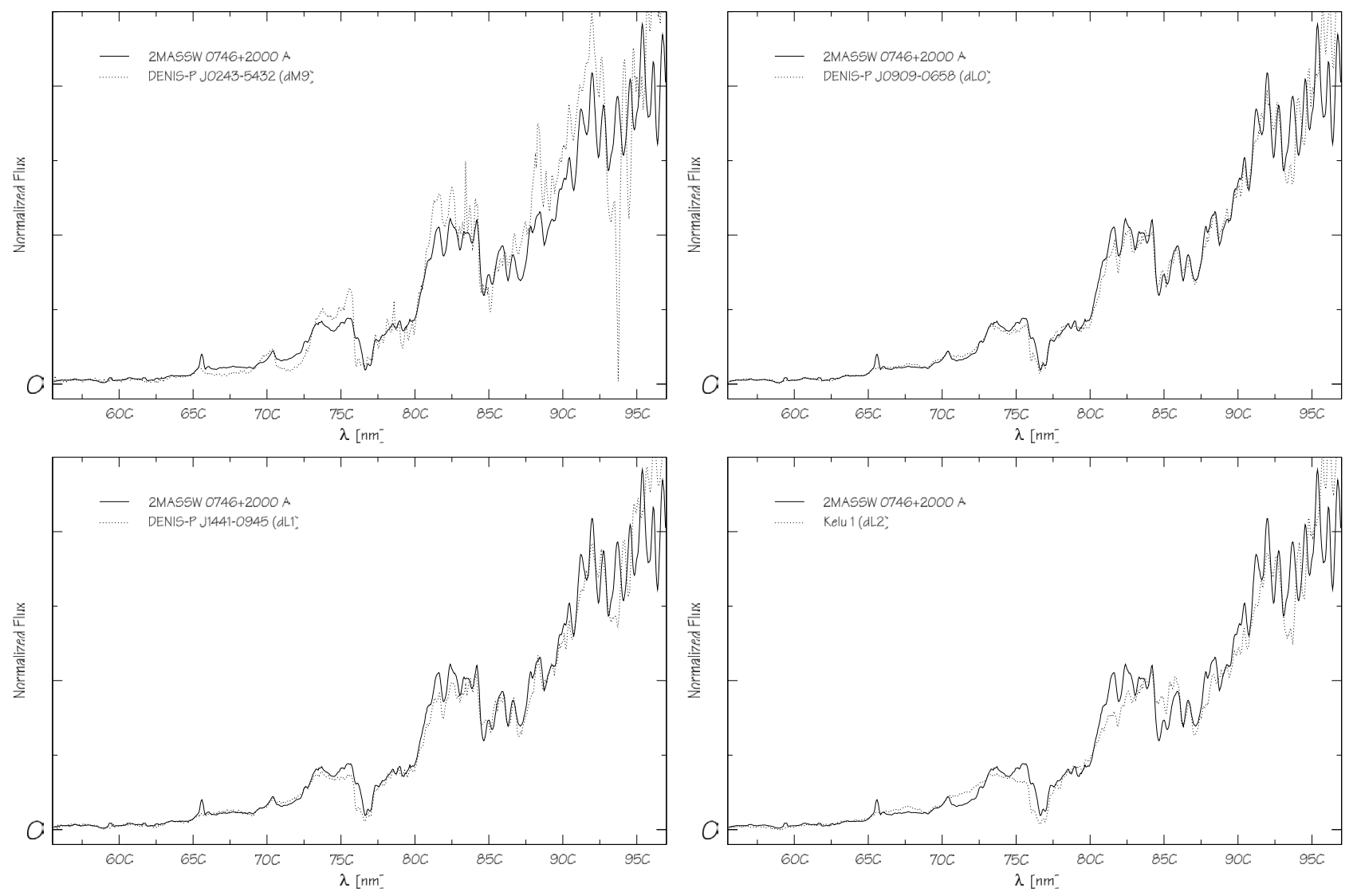

Fig. 5. STIS optical low resolution spectra of 2 MASSW J0746425+2000321A compared to spectra of filed ultra-cool dwarfs. The four plots show the STIS spectrum of 2MASSW J0746425+2000321A, smoothed via a boxcar (width = 5 pixels), and compared to: a) DENIS-P J024351.0-543219 (dM9); b) DENIS-P J090957.1-065806 (dL0); c) DENIS-P J144137.3-094559 (dL1); d) Kelu 1 (dL2). All spectra have been normalized at $840 \mathrm{~nm}$. Spectra for the field dwarfs from Martín et al. (1999).

reduced- $\chi^{2}$ of 1.0 could be argued for, the diversity of the instruments used for this orbital analysis suggests that such a treatment would be at least as erroneous as it could be helpful. For the time being, we decided to stick to the quoted astrometric uncertainties to derive the uncertainties on the orbital parameters of the binary.

\section{Discussion}

\subsection{Spectral types, effective temperatures}

The composite spectrum of 2MASSW J0746425+2000321 AB in the optical has been previously studied by Kirkpatrick et al. (2000), who derived a spectral type of L0.5.

In order to derive the spectral types of each component, we compared their STIS spectra, extracted with the procedure described in Sect. 3.4, with the spectra of field L dwarfs published by Martín et al. (1999). As explained in this latter article, the relative strength of the $\mathrm{TiO}$ bands between 840-860 $\mathrm{nm}$ and the $\mathrm{CrH}$ and $\mathrm{FeH}$ bands between $860-880 \mathrm{~nm}$ are good indicators for the effective temperature changes. In later $\mathrm{L}$ dwarfs the $\mathrm{TiO}$ bands get weaker with respect to the $\mathrm{CrH}$ and $\mathrm{FeH}$ bands. Figures 5 and 6 shows the comparison. 2MASSW J0746425+2000321 A is clearly very similar to the L0 field dwarf DENIS-P J090957.1-065806, while 2MASSW J0746425+2000321 B is between the
L1 (DENIS-P J144137.3-094559) and L2 (Kelu 1) field dwarfs. We thus derive a spectral type of $\mathrm{L} 0 \pm 0.5$ and $\mathrm{L} 1.5 \pm 0.5$ for A and B, respectively. This is consistent with the spectral type obtained by Kirkpatrick et al. (2000), which is a blend of $\mathrm{A}$ and $\mathrm{B}$. It is also consistent with the modest difference in brightness (see Table 3), which implies a difference in temperature of only $100 \mathrm{~K}$ according to the models of Chabrier et al. (2000).

\subsection{Spectral features}

The main emission lines present in the primary's spectrum is $\mathrm{H} \alpha(E W=-25.0 \pm 0.5 \AA, 1-\sigma)$. The spectrum of the secondary is more noisy but the $\mathrm{H} \alpha$ emission line appears clearly, with an equivalent width of $E W=-18.0 \pm 0.5 \AA$. From their high-resolution spectra, Reid et al. (2002) reported a $\mathrm{H} \alpha$ emission of $-1.2 \AA$ for the unresolved system. The difference between the two measurements indicates that 2MASSW J0746425+2000321 A and B display some chromospheric and/or magnetic activity. Li I absorption is not detected in any of the two components with an upper limit of $\sim 1.5 \AA$. Reid et al. (2002) did not detect any Li I absorption with an upper limit of detection at $\leq 0.5 \AA$. The presence of strong resonance doublets of alkali elements (K I at 766.5 and $769,9 \mathrm{~nm} ; \mathrm{Na} \mathrm{I}$ at 818.4 and $819.5 \mathrm{~nm}$; and $\mathrm{Cs} \mathrm{I}$ at 852.1 

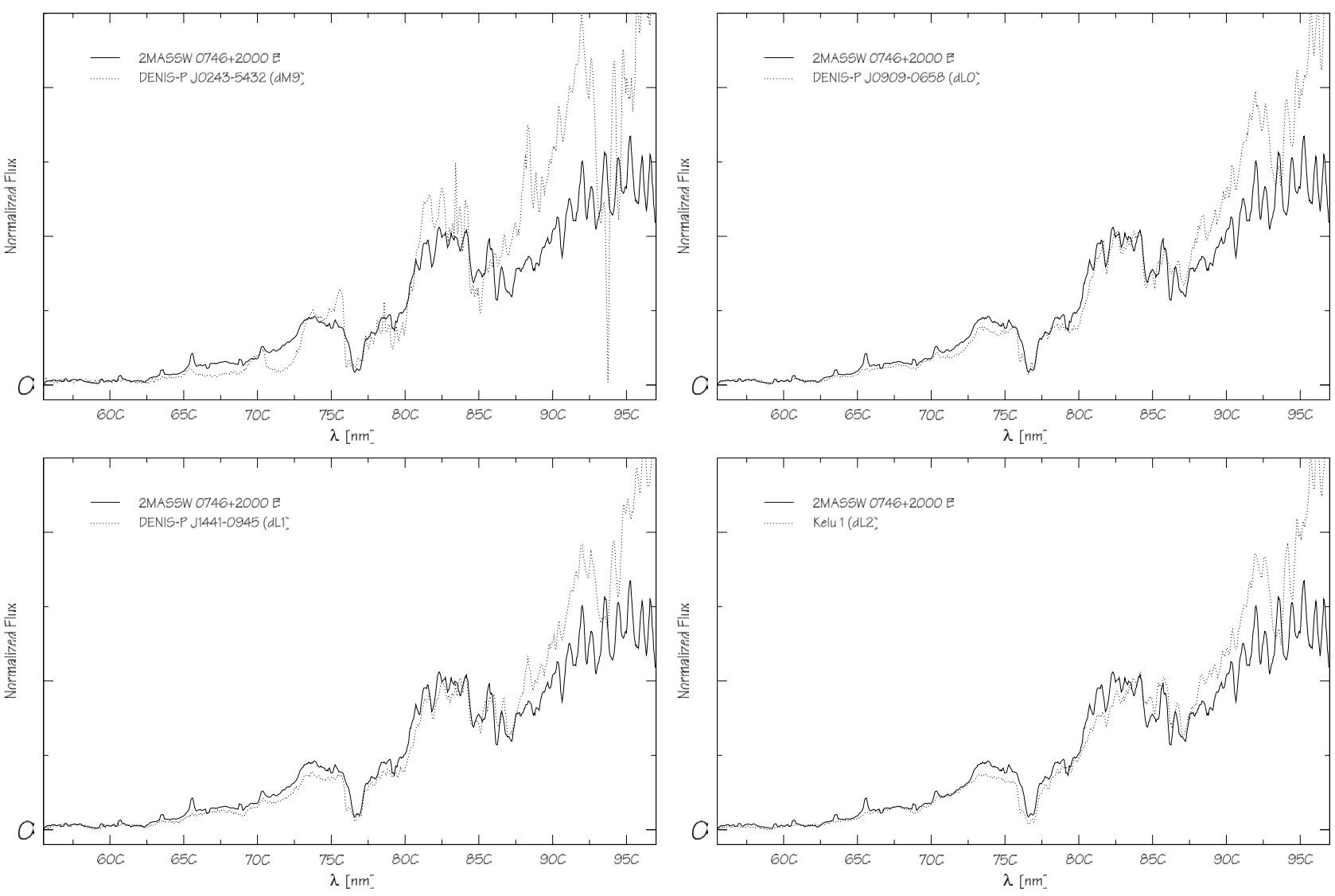

Fig. 6. Same as Fig. 5 but for 2MASSW J0746425+2000321B.

Table 6. Atomic lines in the spectra of 2MASSW J0746425+2000321A and B.

\begin{tabular}{|c|c|c|c|c|c|}
\hline \multirow[b]{2}{*}{ Object } & \multicolumn{2}{|c|}{ K I Wavelength } & \multicolumn{2}{|c|}{ Cs i Wavelength } & \multirow{2}{*}{$\frac{\text { Na I D Wavelength }^{1}}{8183-8195}$} \\
\hline & 7665 & 7699 & 8521 & 8943 & \\
\hline 2MASSW J0746425+2000321A & 22.6 & 17.4 & 2.1 & 0.99 & 8 \\
\hline 2MASSW J0746425+2000321B & 19.0 & 16.4 & $\ldots$ & $\ldots$ & 5 \\
\hline
\end{tabular}

Note. - All units are in angströms. 1- $\sigma$ uncertainties are $\sim 0.5 \AA$.

${ }^{1}$ Corresponds to the blend of the 8183 and $8195 \AA$ doublet.

and $894.3 \mathrm{~nm}$ ) as well as strong metallic molecular band-heads $(\mathrm{CrH}$ at $861.1 \mathrm{~nm}$ and $\mathrm{FeH}$ at $869.2 \mathrm{~nm}$ ) is characteristic for L-dwarfs (Martín et al. 1997). The measurements of equivalent widths of the main atomic lines are reported in Table 6 . It is interesting to note that the equivalent width we measure for Cs I at $852.1 \mathrm{~nm}$ for the primary corresponds to an effective temperature of $\sim 1900-2000 \mathrm{~K}$ in the effective temperature scale of Basri et al. (2000), therefore in good agreement with the effective temperature derived by Schweitzer et al. (2001) from their comparison of low and high resolution Keck spectra with the DUSTY models.

\section{3. colour-magnitude diagrams}

Figure 7 shows a colour-magnitude diagram of 2MASSW J0746425+2000321 AB, 2MASSW J0746425$+2000321 \mathrm{~A}$ and 2MASSW J0746425+2000321 B, and compares with the isochrones of the most recent DUSTY models for solar metallicity. To convert the observed magnitudes to absolute magnitudes we used the trigonometric parallax reported by Dahn et al. (2002).

The position of 2MASSW J0746425+2000321A shows that the age ranges between 150 and $500 \mathrm{Myr}$, thus relatively young. This is not consistent with the very high surface gravity obtained by Schweitzer et al. (2001). They used high and low resolution unresolved spectra and compared it with the DUSTY atmospheric models of Allard et al. (2001). Using a $\chi^{2}$-fitting algorithm, they obtain an effective temperature of $2000 \mathrm{~K}$ and a surface gravity $\log g=6.0$ from their low resolution spectra. From their high resolution spectra, they obtain an effective temperature of $1900 \sim 2000 \mathrm{~K}$ and a surface gravity $\log g=5.0 \sim 5.5$. The temperatures are in good agreement with the spectral types and colours we report here, but the surface gravity is too high for the young age. This is probably 


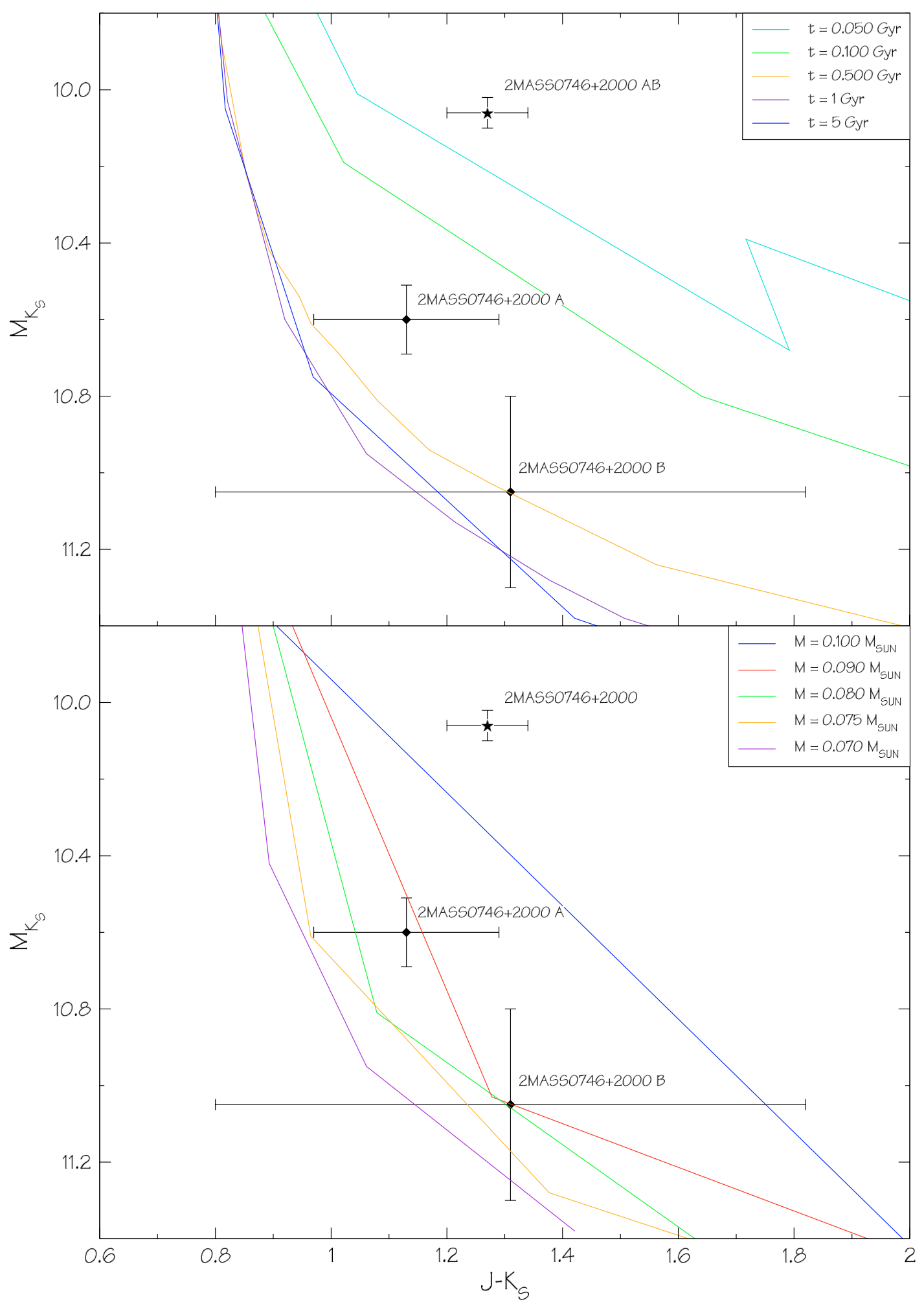

Fig. 7. Colour-magnitude diagrams $M_{K_{\mathrm{S}}}$ vs. $\left(J-K_{\mathrm{S}}\right.$ ) displaying the location of $2 \mathrm{MASSW}$ J0746425+2000321A, B and AB (combined light). The $1-\sigma$ combined uncertainties include the uncertainty on the distance. Isochrones of the DUSTY models (Chabrier et al. 2000) are overplotted for different ages (upper panel) and different masses (lower panel).

because the DUSTY models overestimate the dust effects. As a consequence, the strength of the alkali lines in the optical decreases, and the surface gravity is biased toward higher values.

The mass of the primary ranges between 0.075 and $0.095 M_{\odot}$, while the mass of the secondary has large error bars and ranges between 0.055 and $0.100 M_{\odot}$. The total mass of the system therefore ranges between $\sim 0.130$ and $\sim 0.190 M_{\odot}$, which is consistent with the dynamical mass considering the large uncertainties in the H-R diagram. 
For the secondary's mass it appears more appropriate to use the mass of the primary from the H-R diagram, which has reasonable uncertainties, together with the very precise dynamical total mass. This yields a mass between 0.052 and $0.072 M_{\odot}$, therefore clearly substellar. The absence of lithium absorption in the spectra gives also a constrain on the lower limit of the mass. According to the DUSTY evolutionary models, Lithium should be depleted for masses greater than $0.075 M_{\odot}$ at $150 \mathrm{Myr}$ and masses greater than $0.060 M_{\odot}$ at $500 \mathrm{Myr}$. The mass of the secondary must therefore be greater than 0.060 , and ranges between $0.060 \leq M_{B} \leq 0.072 M_{\odot}$.

The system is thus very likely made of a brown dwarf orbiting a slightly more massive very low mass star. Both objects are very close to the stellar-substellar boundary.

\section{Conclusion}

We present new astrometric, photometric and spectroscopic observations of the ultra-cool 2MASSW J0746425+2000321AB pair, which have enabled us to compute the orbital parameters, total and individual masses and spectral types of the system. We find a total mass of $0.146_{-0.006}^{+0.016} M_{\odot}(2-\sigma)$, with a $\mathrm{L} 0 \pm 0.5$ primary and a $\mathrm{L} 1.5 \pm 0.5$ secondary. The orbit is eccentric $\left(e=0.41_{-0.09}^{+0.08}\right)$ with a period of $3850.9_{-767}^{+904}$ days. Our observations enable us to follow $60 \%$ of the orbit, corresponding to $36 \%$ of the period. Near-infrared photometry of the individual components enables us to locate them in a H-R diagram and compare them to the most recent evolutionary DUSTY models. The pair is likely formed by a brown dwarf (L1.5 \pm 0.5 , with $0.060 \leq M_{B} \leq 0.072 M_{\odot}$ ) orbiting a $\mathrm{L} 0 \pm 0.5$ very low mass star $\left(0.075 \leq M \leq 0.095 M_{\odot}\right)$. The system appears to be young, with an age in the range $150 \sim 500$ Myr. Further high-angular resolution images should allow to cover $100 \%$ of the orbit and refine the orbital parameters. Radial velocity measurements, if feasible, would then allow to compute precise individual masses.

Acknowledgements. The authors thank the referee of this manuscript, Dr M. Simon for the useful comments and advices he made on this paper. This work is based on observations collected at the European Southern Observatory (Paranal, Chile), program 70.D-0773; with the NASA/ESA Hubble Space Telescope obtained at the Space Telescope Science Institute (STSci), programs GO8720 and GO9451, and at the Keck Observatory (Hawai'i, USA). We would like to acknowledge the great cultural significance of Mauna Kea for native Hawaiians, and express our gratitude for permission to observe from its summit. The STSci is operated by the Association of Universities for Research in Astronomy, Inc., under NASA contract NAS 5-26555. This publication makes use of data products from the Two Micron All Sky Survey, which is a joint project of the University of Massachusetts and the Infrared Processing and Analysis Center/California Institute of Technology, funded by the National Aeronautics and Space Administration and the National Science Foundation. This work made use of data from the The Guide Star Catalogue II, which is a joint project of the Space Telescope Science Institute and the Osservatorio Astronomico di Torino. The participation of the Osservatorio Astronomico di Torino is supported by the Italian Council for Research in Astronomy. Additional support is provided by European Southern Observatory, Space Telescope European Coordinating Facility, the International GEMINI project and the European Space Agency Astrophysics Division.

\section{References}

Allard, F., Hauschildt, P. H., Alexander, D. R., Tamanai, A., \& Schweitzer, A. 2001, ApJ, 556, 357

Bailer-Jones, C. 2004 [arXiv: astro-ph/0403013]

Basri, G., Mohanty, S., Allard, F., et al. 2000, ApJ, 538, 363

Bouy, H. 2004, in prep.

Bouy, H., Brandner, W., Martín, E. L., et al. 2003, AJ, 126, 1526

Brandner, W., Rousset, G., Lenzen, R., et al. 2002, The Messenger, 107

Burrows, A., Marley, M., Hubbard, W. B., et al. 1997, ApJ, 491, 856

Chabrier, G., Baraffe, I., Allard, F., \& Hauschildt, P. 2000, ApJ, 542, 464

Clarke, F. J., Oppenheimer, B. R., \& Tinney, C. G. 2002, MNRAS, 335,1158

Close, L. M., Siegler, N., Freed, M., \& Biller, B. 2003, ApJ, 587, 407

Cutri, R. M., Skrutskie, M. F., van Dyk, S., et al. 2003, VizieR Online Data Catalog, 2246, 0

Dahn, C. C., Harris, H. C., Vrba, F. J., et al. 2002, AJ, 124, 1170

Forveille, T., Beuzit, J., Delfosse, X., et al. 1999, A\&A, 351, 619

Gelino, C. R., Marley, M. S., Holtzman, J. A., Ackerman, A. S., \& Lodders, K. 2002, ApJ, 577, 433

Ghez, A. 1993, Ph.D. Thesis

Kim Quijano, J., Mobasher, B., Brown, B., et al. 2003, Baltimore: STScI

Kirkpatrick, J. D., Reid, I. N., Liebert, J., et al. 2000, AJ, 120, 447

Kleinmann, S. G., Lysaght, M. G., Pughe, W. L., et al. 1994, Experimental Astron., 3, 65

Lane, B. F., Zapatero Osorio, M. R., Britton, M. C., Martín, E. L., \& Kulkarni, S. R. 2001, ApJ, 560, 390

Leggett, S. K., Golimowski, D. A., Fan, X., et al. 2002, ApJ, 564, 452

Lenzen, R., Hartung, M., Brandner, W., et al. 2003, in Instrument Design and Performance for Optical/Infrared Ground-based Telescopes, ed. Iye, Masanori; Moorwood, Alan F. M., Proc. SPIE, 4841, 944

Marquardt, D. W. 1963, J. Soc. Industr. Appl. Math., 11, 431

Martín, E. L., Basri, G., Delfosse, X., \& Forveille, T. 1997, A\&A, 327, L29

Martín, E. L., Delfosse, X., Basri, G., et al. 1999, AJ, 118, 2466

Matthews, K., Ghez, A. M., Weinberger, A. J., \& Neugebauer, G. 1996, PASP, 108, 615

Monet, D. G., Levine, S. E., Canzian, B., et al. 2003, AJ, 125, 984

Patience, J., Ghez, A. M., Reid, I. N., Weinberger, A. J., \& Matthews, K. 1998, AJ, 115, 1972

Pavlovsky, C., Biretta, J., Boffi, F., et al. 2003, Baltimore: STScI

Press, W. H., Teukolsky, S. A., Vetterling, W. T., \& Flannery, B. P. 1992, Numerical recipes in C. The art of scientific computing (Cambridge: University Press, 2nd ed.)

Reid, I. N., Gizis, J. E., Kirkpatrick, J. D., \& Koerner, D. W. 2001, AJ, 121, 489

Reid, I. N., Kirkpatrick, J. D., Gizis, J. E., et al. 2000, AJ, 119, 369

Reid, I. N., Kirkpatrick, J. D., Liebert, J., et al. 2002, AJ, 124, 519

Rousset, G., Lacombe, F., Puget, P., et al. 2003, in Adaptive Optical System Technologies II, ed. P. L. Wizinowich, \& D. Bonaccini, Proc. SPIE, 4839, 140

Schweitzer, A., Gizis, J. E., Hauschildt, P. H., Allard, F., \& Reid, I. N. 2001, ApJ, 555, 368 